\title{
LAB-ON-A-CARD ASSAY FOR ENTERIC PATHOGENS
}

B. H. Weigl (1)*, J. Gerdes (2), P. Tarr (4), P. Yager (3), L. Dillman (1), J. Gerlach (1), M. Steele (1), R. Peck (1), S. Ramachandran (3), M. Lemba (3), D. Hoekstra (2), M. Kokoris (2), M. Nabavi (2), F. Battrell (2), D. M. Denno (3), E. J. Klein (3)

(1) Program for Appropriate Technology in Health (PATH), 1455 NW Leary Way, Seattle, WA 98107, Telephone (206) 285-3500, FAX (206) 285-6619; (2) Micronics, Inc., Redmond, WA; (3) University of Washington, Seattle, WA; and (4) Washington University, St. Louis, MO.

\begin{abstract}
We describe the functional elements of a diagnostic instrument and disposable enteric card (DEC) system under development that rapidly identifies and differentiates Shigella dysenteriae serotype 1, Escherichia coli O157:H7, Campylobacter jejuni, and Salmonella and Shigella species in stool samples. These elements, currently realized as microfluidic subcircuits on individual cards, are (1) whole pathogen immunocapture, (2) nucleic acid extraction, (3) on-chip rapid PCR, and (4) lateral flow detection of amplicons. The system utilizes a low-cost disposable lab-on-a-card platform designed to identify enteric bacterial pathogens in patients with acute diarrhea. Special emphasis is placed on the utility of the device to diagnose both intentionally released enteric biothreat agents as well as to provide a platform to identify infections that are common worldwide. All reagents are stored in dry form on the card.

In this paper we report on the design and validation of individual subcircuits, identification and validation of capture antibodies and strategy for organism immunocapture, and identification and validation of specific PCR primer sequences for over 200 clinical isolates of enteric pathogens as well as spiked and pathogenic stool samples.
\end{abstract}

\section{INTRODUCTION}

Bioterrorist use of enteric pathogens is a significant potential threat, and naturally occurring enteric infections already burden global health. Enteric disease can result from bacterial, viral, or protozoal contamination of food and water, and infectious diarrhea is the second leading cause of morbidity and mortality worldwideaccounting for an estimated 3,100,000 deaths annually. In the United States, the rate of infectious diarrhea is lower than in developing countries, but it is estimated that $375,000,000$ cases of diarrhea occur in the U.S. annually resulting in $73,000,000$ physician consultations, 1,800,000 hospitalizations, and 3,100 deaths [1-3]. The cost of medical care and lost productivity from infectious diarrhea in the U.S. is estimated at $\$ 6$ to $\$ 23$ billion.

The intentional contamination of a salad bar with Salmonella [4] and a laboratory coworker's food with Shigella stolen from the laboratory [5] are two examples of bioterrorism on US soil. In both cases, an enteric pathogen was effectively being used as a biothreat agent.

Diagnosing the pathogen that causes diarrhea is a nontrivial undertaking under the best conditions, and not feasible when resources are extremely limited as in resource-poor countries, or if developed systems are stressed during a biothreat crisis. Syndromic management of diarrhea without any laboratory information for the patient and/or health care worker is not ideal, yet it is a reality in many settings. Where available, culture methods are used to detect and identify organisms that may be present, but the application of these methods is sporadic, time consuming, expensive, and lacks specificity. Technology in clinical laboratory identification of enteric pathogens has changed little since the early 1900 s, and utilizes a series of culture plates with selective media to isolate and culture target organisms (Figure 1). This process is consumes time, requires skilled labor, and is costly. C. jejuni and E. coli O157:H7 require typically 1 to 2 days from receipt of specimen to result, while Salmonella and Shigella require between 2 and 4 days.

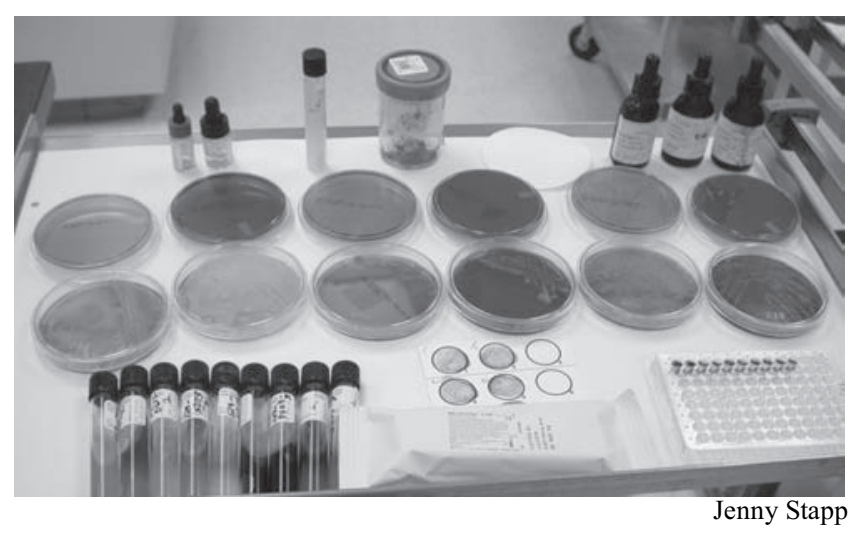

Figure 1. Culture plates representing a thorough clinical workup including Aeromonas, Campylobacte, E. coli O157:H, nonO157:H7 STEC, Plesiomonas, Salmonellae, Shigellae, Vibrios, Yersiniae, at a cost of $\sim$ US\$199.

Culture methods to detect $E$. coli $\mathrm{O} 157: \mathrm{H} 7$ generally utilize selective media based upon the observation that E. coli O157:H7 do not ferment sorbitol. However, other enteric bacteria can share this phenotype, and there are reports of sorbitol-fermenting, toxigenic and pathogenic E. coli O157 that are fairly common in Europe [6]. Screening that is based solely on the sorbitol phenotype may therefore result in falsely negative results. Furthermore, confirmatory testing is required for sorbitol nonfermentors utilizing additional resources and time. Excellent results can be obtained retrospectively with complete bacteriological characterization, but it is a technology that is too slow for practical use in response to an outbreak or in clinical situations.

Other identification techniques include enzyme immunoassays (EIAs) that detect organism antigens or their toxins and genotyping. Tu et al. [7] reported an EIA that detects E. coli O157:H7. Recently, a triplex PCR assay for the detection of Campylobacter jejuni, Salmonella spp., and Escherichia coli O157:H7 was reported [8] that used genomic identification of the bacteria but was not specific to virulence factors associated with these pathogens.

The importance of all four of these agents (Shigella dysenteriae type 1 (Sd1), STEC, C. jejuni, and Salmonella spp.) is demonstrated by their classification as Category B priority pathogens based on NIAID Biodefense Research [9]. In addition, with the possible exception of E. coli $0157: \mathrm{H} 7$, these agents are common pathogens in many developing settings.

$\mathrm{Sd} 1$ is an especially virulent pathogen that causes regional outbreaks of dysentery. Symptoms of cramps, diarrhea, blood or pus in the stool, fever, and/or vomiting can begin in as few as 12 hours after ingestion. Sd1 is a plausible bioterrorism agent because of its virulence, highly infectious nature, and hardiness in the 
environment. An infectious dose can be as few as 10 organisms. During infection, Sd1 invades and destroys the cells that line the large intestine and is very prolific-shedding one million to one hundred million organisms per gram of stool. Without prompt and effective treatment, the fatality rate of $\mathrm{Sd} 1 \mathrm{can}$ be as high as $15 \%$.

E. coli $\mathrm{O} 157: \mathrm{H} 7$ produces one or more toxins similar to $\mathrm{Sd} 1$, which often leads to hemorrhagic colitis. Like Sd1, STEC disproportionately affect the young and elderly. Symptoms include crampy abdominal pain, vomiting, and watery diarrhea, which usually progresses to bloody diarrhea. While STEC infections are self-limiting, up to $10 \%$ of patients will develop hemolytic uremic syndrome (HUS) [10]. It is estimated that there is an overall mortality rate of 1 to 35 percent among patients infected with STEC. E. coli O157:H7 is the most commonly occurring STEC and in 2001, 3,478 cases of STEC were reported in the U.S. alone.

Campylobacter jejuni is the leading cause of bacterial diarrhea in the U.S. with an estimated number of cases exceeding four million annually [10]. Symptoms include fever, nausea, muscle pain, and watery diarrhea that may contain blood. Most infections are self-limiting, but complications, especially in susceptible populations, may include reactive arthritis, septicemia, and Guillain-Barré Syndrome.

Salmonella species are responsible for two to four million cases of diarrhea in the U.S. each year [11]. Salmonella has previously been used as a bioterrorism agent. In 1984, a religious cult in Oregon poisoned salad bars with Salmonella causing disease in 751 people [4].

\section{THEORY}

The multiplex DEC test we are developing (see schematic in Figure 2) will be an automated, easy-to-use, point-of-care platform to detect simultaneously and rapidly, multiple different enteric

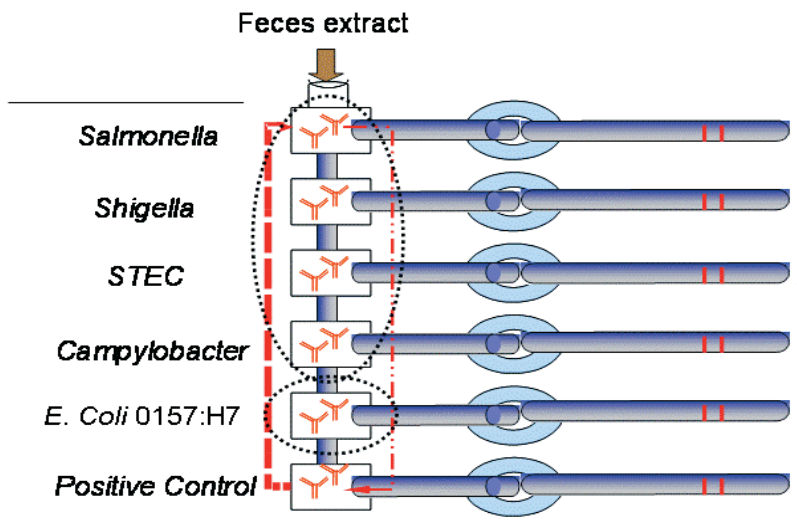

Immunocapture NA amplification NA LFS detection

Figure 2. Schematic of DEC approach. Showing a combination of whole pathogen immunocapture (immunocapture agents for Salmonella, Shigella, and Campylobacter are grouped together), nucleic acid extraction, PCR, and lateral flow detection of amplicons.

pathogens that cause disease with similar symptoms, ie. diarrhea. The method is unique in that it seeks the bacterial agent using a orthogonal approach via antigen capture and the amplification of specific virulence genes for precise identification of the specific etiological agent. It will provide a mechanism for accurate, pointof-care diagnosis with a rapid turn-around time for results. The method is based on microfluidic lab card technology developed at
Micronics and the University of Washington. This technology has been used in many different applications ranging from diffusionbased separation and detection [12-17] to projects involving cytometry on a chip [18-21] and nucleic acid-based amplification and detection techniques [22].

\section{EXPERIMENTAL DETAILS}

Presently, we have implemented each of the four elements of the DEC as microfluidic subcircuits on individual cards before embarking on full integration of the disposable device. Fluid transfer between the subcircuits occurs by pipette. The subcircuits are (1) whole pathogen immunocapture, (2) nucleic acid extraction, (3) on-card rapid PCR, and (4) lateral flow detection of amplicons, and are described below. In addition, we have demonstrated the feasibility of drying and resuspending all reagents needed for the DEC, thus allowing the possibility of storage at ambient temperature.

(1) Immunocapture of whole organisms: Our approach to capture pathogens on the DEC is based on antibodies coupled to magnetic beads. Figure 3 shows the current immunocapture subcircuit.

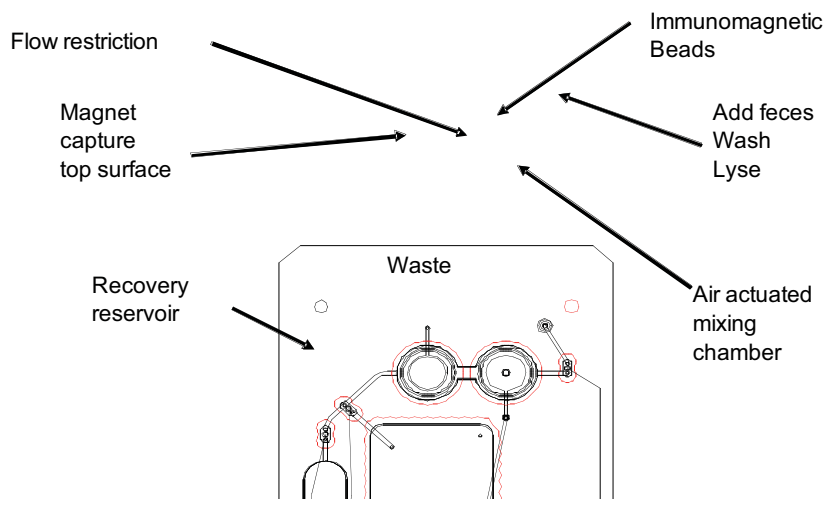

Figure 3. Whole pathogen immunomagnetic bead capture subcircuit.

One (or more in most cases) antibody suitable for whole organism capture has been identified for each target pathogens and validated using EIA plates, a magnetic bead-based capture system, or both. Organism capture has been validated from spiked stool for E. coli O157:H7, Salmonella, and C. jejuni. In addition, freshly shed stools containing $E$. coli $\mathrm{O} 157: \mathrm{H} 7$ have also been tested and were positive. Further, E. coli $\mathrm{O} 157: \mathrm{H} 7$ is especially difficult to identify by nonmultiplexed PCR. Therefore, we cultured and tested 14 confounder organisms (selected on a phylogenetic basis, to increase the validity of the results) with the selected antibody to determine that only pathogenic strains are captured. Additionally, three microfluidic subcircuits (the nucleic acid extraction, PCR amplification, and a qualitative lateral flow detection subcircuit), have been completed and tested. PCR primers were designed for all organisms and their specificity confirmed. 200 clinical isolates were cultured and tested with PCR to validate primer specificity. In addition a fourth subcircuit, the antibody capture subcircuit, has been validated for feces in a tube format and is currently being adapted to a lab card.

One significant technical accomplishment of this program is its validation of the selectivity of antibody for whole organism for direct capture from both frozen and fresh naturally infected human 
stool, and spiked normal stools. It was unclear at the beginning of this project if sufficient selectivity could be achieved in such a microbially complex milieu and if the target organisms of interest reliably express the critical surface antigens during infection. However for all spiked and fresh stool samples so far tested, the capture methodology was concordant with the EIA and culture results. Another significant finding relates to the combined selectivity approach provided by immunocapture and PCR. After completing primer design and validation for all organisms, and selecting and validating capture antibodies, we found that primers for all target organisms except for E. coli O157:H7 are sufficiently specific to amplify the target sequence in the presence of other organisms. On the other hand, we found that the capture antibody for $E$. coli $\mathrm{O} 157: \mathrm{H} 7$ is quite specific in the presence of other target organisms, as well as in fresh and spiked stool samples. Thus, antibodies can be used to partially purify E. coli O157:H7 from generic E. coli and other flora in stool.

(2) Nucleic acid (NA) extraction on microfluidic card: A card that can purify RNA from lysed leukocytes or bacteria (Figure 4) is loaded with specimen in a lysis solution which contains a chaotropic buffer that allows RNA from the sample to bind to silica. On-card silica filter and microfluidic valves for fluid control and automate RNA binding, washing, drying, and elution. The card was initially validated in experiments in which we processed $10^{6}$ white blood cells using commercial kits or suspended in lysis buffer, before applying samples to the port on the lab card. LightCycler assays determined that the microfluidic card solutions had a slightly lower crossing point (25.3) than the control RNeasy solutions (27.1). This card also has been validated for lysis and detection of Gram-negative bacteria in feces.

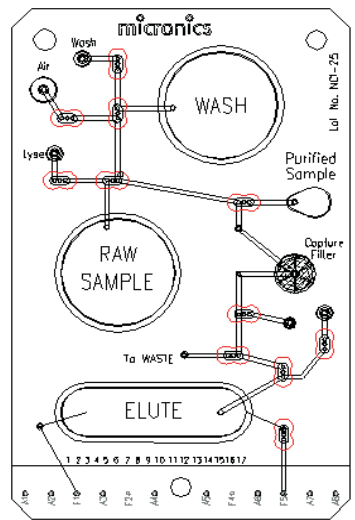

Figure 4. Schematic of nucleic acid extraction subcircuit. The operational steps currently are:

(1) sample injected

(2) nucleic acids captured on silica membrane

(3) wash

(4) dry membrane

(5) elute

(6) eluent taken for analysis

The purification of nucleic acid on the card is completed in $<5$ minutes and is under complete software control.

3) PCR subcircuit: Micronics has developed a prototype thermal control unit (TCU) for extremely rapid temperature ramps (Figure 5). This TCU thermocycles the amplification chamber of a disposable microfluidic card. For efficient thermal transfer, a simple laminate card was designed with an amplification chamber capped by one layer of 0.004" Mylar, permitting the capping layer of the chamber to touch the TCU. The 16-second cycle time (longest in tests) can be diminished considerably with heat sinks, TCUs, and efficient thermal interface material. Even at 16 seconds, 30 full cycles requires only 8 minutes.

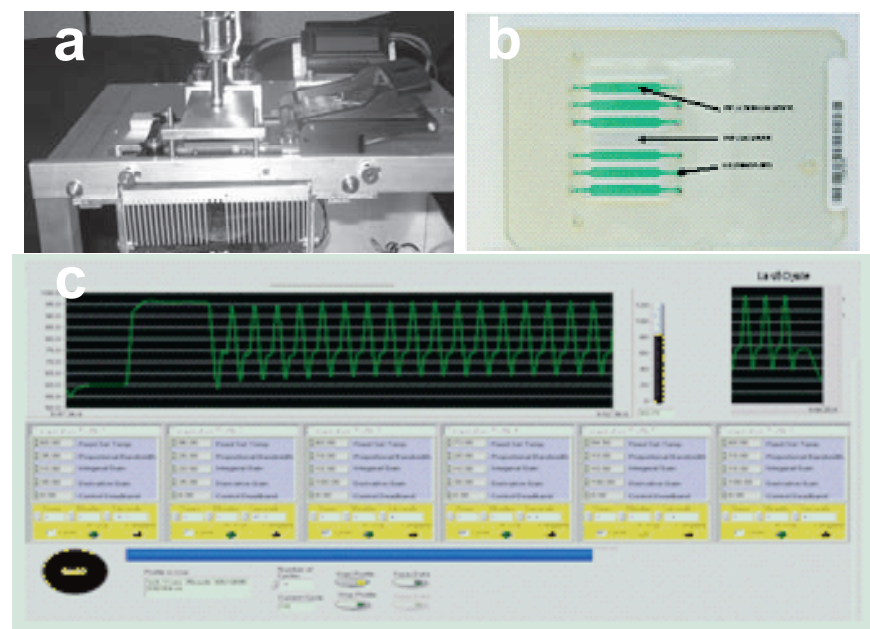

Figure 5. 5A Micronics-developed TCU. 5B PCR amplification lab card capable of performing six amplifications simultaneously, $5 \mathrm{C}$ and software interface with thermal couple trace showing 60 second $60^{\circ} \mathrm{C}$ reverse transcription followed by 16 -second PCR cycles.

Figure 6 demonstrates the fluid control system that operates both on-card valves (using a small air pump) and fluid control (using positive displacement pumps).

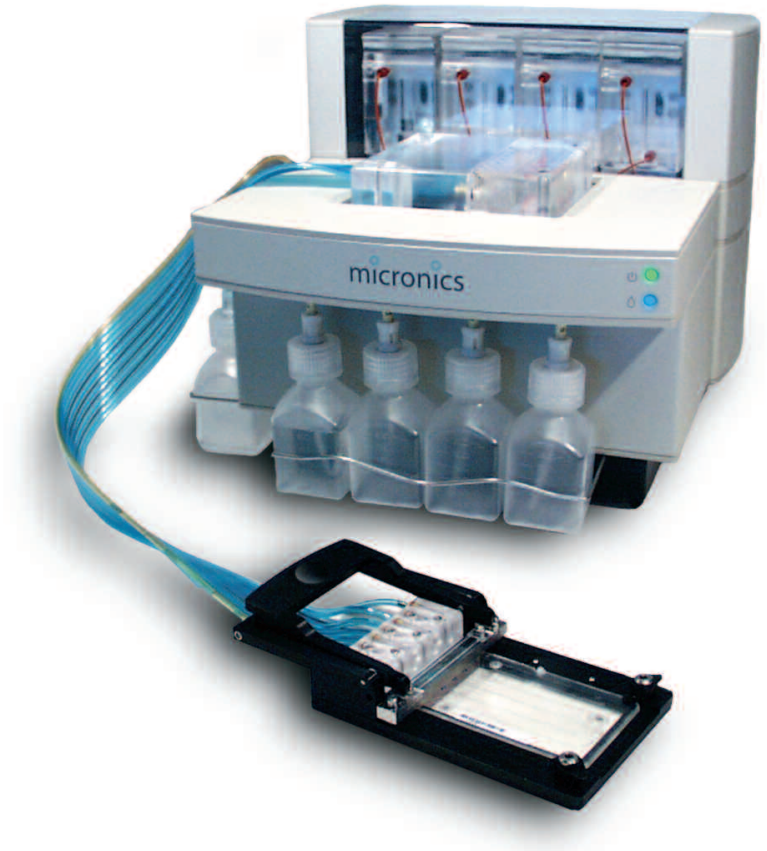

Figure 6. DEC Instrument and disposable interface-current prototype (shown without thermocycler attachment).

Figure 7 exemplifies the products of PCR subcircuit, with a gel showing the products of extracted and purified Salmonella. Similar results have been obtained with all target organisms. In the case E. coli $0157: \mathrm{H7}$, we have applied this system to human stool from children infected with this pathogen. 


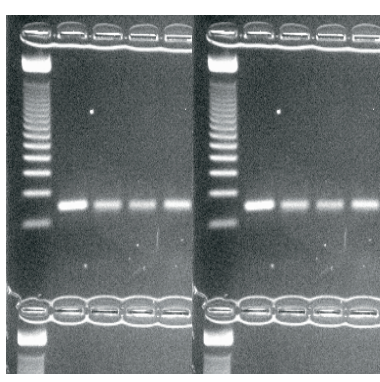

Figure 7. Ethidium bromide stained DNA in agarose gel of salmonella amplified with the DEC PCR subcircuit. The left lane for the upper and lower gel is a MW marker; lane 1 for each gel is the result from a standard thermal cycler; the other 16 lanes are results for the DEC PCR subcircuit run for 15 minute and 34 cycles.

(4) Lateral flow (LF) detection of amplicons: For this project, we are adapting an existing endpoint detection system using a LF amplicon detection method. This technique allows nonelectrophoretic detection of PCR products, and obviates the use of gels or sophisticated optics to measure dye-coupled amplicons.

Figure 8 illustrates the components in the LF strips and how the reactions are created. In brief, amplicons (the bar with $5^{\prime}$ and $3^{\prime}$ ends) bind to dried capture probes to create detection probes nested within the amplicon. One capture probe has a biotin label and the other has a fluorescein (FITC) label (used here as a hapten). As the solution wicks along the strip, streptavidin-coated microparticles move with the solution, and a streptavidin/biotin association is formed between microparticles and the amplicon/capture duplex. As they migrate through the LF strip, the microparticles with detection probes bind to a previously deposited line of anti-FITC antibodies through the FITC hapten on the other capture probe. As the chromogenic particles accumulate on the antibody stripe, a blue line becomes apparent, indicating successful NA amplification and the presence of target. Procedural control lines have been included, and results are available in a few minutes.

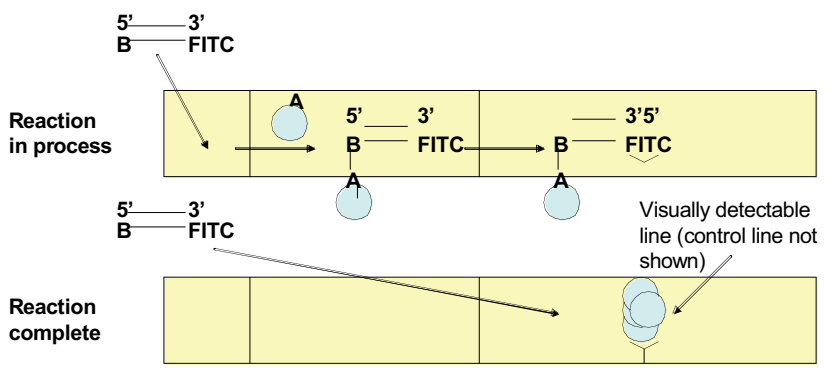

Figure 8: Schematic of amplicon detection using lateral flow strips. The strips operate by simple wicking (no incubations or washes are needed) and will be integrated on the DEC.

Figure 9 demonstrates amplicons with the lateral flow strip, showing PCR products from the amplified Salmonella

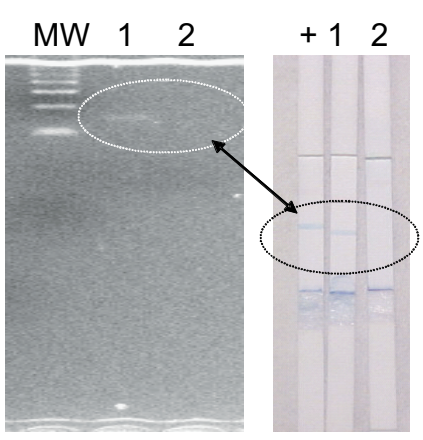

5) Dry Reagent Storage: On-card dry reagent storage will enable the use of the DEC system without refrigeration for reagents and disposables. In addition, it will remove the need for external, bulky, liquid reagents (except for stable aqueous resuspension and driving fluid resevoirs). To determine the feasibility of the reagent drydown and resuspension process, three different reagent classes have been studied: 1) antibody coated magnetic beads for pathogen capture, 2) lysis buffer for extraction of nucleic acids from the pathogens, and 3) amplification of DNA by polymerase chain reaction (PCR).

Several methods to stabilize proteins already exist and are widely used in industry. These methods use trehalose, a nonreducing disaccharide, which forms a glassy state above room temperature. The protein is protected against degradation and crystallization because low molecular mobility in the glassy state [23]. The native state of the protein is thought to be maintained largely because of the substitution of the waters of hydration of protein by sugar molecules during drying [24]. However all these methods use freezedrying. A novel method to control dissolution of preserved biomolecules in a microfluidic device has been previously studied in which enzyme is preserved in a trehalosedextran matrix at elevated temperatures.

The preservation method has been tested for the reagents for immunocapture, lysis, and for the PCR master mix.

Bead preservation: Tosyl-activated magnetic beads of $1 \mu \mathrm{m}$ diameter (Dynal Biotech LLC) were covalently linked with antibodies to E. coli (Abcam) according to the supplier's instructions. The beads were suspended in buffer containing varying concentrations of trehalose $(5-20 \% \mathrm{w} / \mathrm{v})$ and trehalosedextran (1:1). Ca. $0.1 \mathrm{mg}$ of beads in $20 \mu \mathrm{L}$ of volume were spotted on a Mylar membrane and dried in an oven at $37^{\circ} \mathrm{C}$ under $15-18 \%$ humidity conditions. Resuspension was studied in a flow channel constructed with Mylar around the bead spot. Antibodycoated magnetic beads retained their ability to capture $E$. coli after resuspension from dehydrated state. The beads aggregated as is evidenced by SYTO 9 staining of the bacteria (Figure 10).
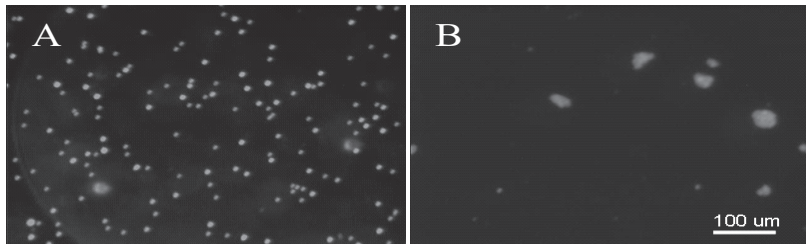

Figure 10. 10a shows magnetic beads after resuspension from dehydrated state. $\mathbf{1 0 b}$ shows SYTO 9-stained E. coli captured by the magnetic beads.

Lysis buffer preservation: The buffer reagent for lysis of the pathogens as part of the nucleic acid extraction subcircuit does not contain molecules that need protection from harsh conditions. However, in the dry form the lysis buffer crystallizes, which may be undesirable for incorporation into small channels of a microfluidic device. The addition of trehalose formed a matrix; but only $40 \%$ trehalose concentration produced a nearly complete glassy matrix (Figure 11).

Lysis buffer containing $4.5 \mathrm{M}$ guanidinium thiocyanate, 50 $\mathrm{mM}$ MES, pH 5.5, $20 \mathrm{mM}$ EDTA, $1 \% \mathrm{~N}$ lauroyl sarcosine and 5 $\%$ Triton X100 were prepared containing varying amount of trehalose $(0-40 \% \mathrm{w} / \mathrm{v})$. About $10 \mu \mathrm{l}$ of buffer were spotted on to Mylar membrane and dried $\left(37^{\circ} \mathrm{C}, 15-18 \%\right.$ humidity $)$. 


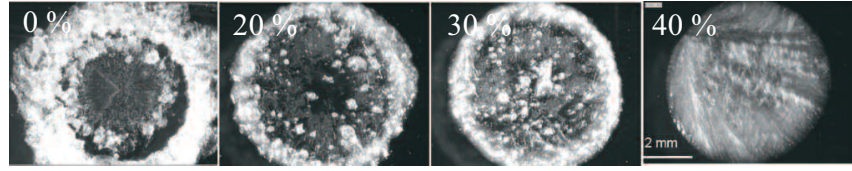

Figure 11. Shows stereomicroscope images of dehydrated spots of lysis buffer in varying concentration of trehalose ( $w / v)$.

Resuspension of the dehydrated buffer demonstrated no difference in the presence or absence of trehalose. The crystals readily re-solubilize, and the buffer successfully lysed the bacteria. No colony-forming units were detected on agar plates.

PCR master-mix preservation: PCR master mix (ThermoScript ${ }^{\mathrm{TM}}$ Plus Platinum Taq mix from Invitrogen) containing dNTPs, polymerase enzyme, Salmonella Primer mix (Vanilla) and buffer were prepared and dissolved in equal volume of varying concentration of trehalose to give a final concentration of $0,10,15$ and $20 \%(\mathrm{w} / \mathrm{v})$. The master mix was pipetted into polystyrene wells of a 96-well plate and dried $\left(37^{\circ} \mathrm{C}, 15 \%\right.$ humidity) for varying intervals. After drying, the master mix was rehydrated with water. Ca. 1 x $10^{5} \mathrm{CFU} / \mu \mathrm{L}$ DNA template (Salmonella) was added and the sample loaded on to the PCR subcircuit card. PCR was performed using the TEC prototype. After an initial denaturation at $90^{\circ} \mathrm{C}$ for $1.0 \mathrm{~min}, 35$ cycles of amplification were performed with denaturation $\left(90^{\circ} \mathrm{C} 8 \mathrm{~s}\right)$, annealing $\left(50^{\circ} \mathrm{C}\right.$ for $\left.10 \mathrm{~s}\right)$ and elongation $\left(6{ }^{\circ} \mathrm{C}\right.$ for $\left.12 \mathrm{~s}\right)$. Amplified DNA was electrophoresed in 3\% agarose, ethidium-stained, and viewed under UV illumination.

The PCR master mixes retained their activity after storage in a trehalose matrix (Figure 11). A $70 \mathrm{bp}$ amplicon was detected after PCR of the resuspended samples. Absence of trehalose resulted in loss of enzyme activity within $24 \mathrm{~h}$ and formation of primer-dimers. The functionality of the master mix could be preserved even after 28 days.

Figure 12. Ethidium bromide-stained DNA in agarose gel after PCR of trehalose preserved master-mix. 12A shows a $70 \mathrm{bp}$ amplicon in lane 2 (positive control) and corresponding amplicon

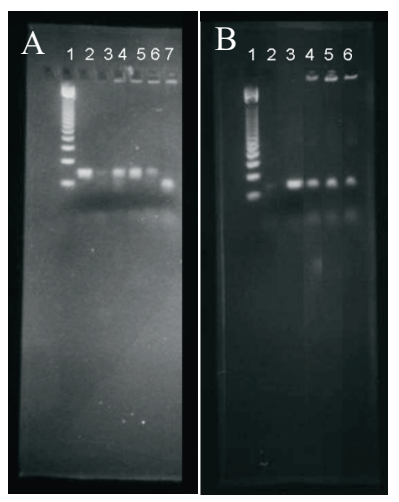
in lane 4-5 of samples preserved in 10, 15 and 20\% trehalose respectively for $24 \mathrm{~h}$. Lane 7: sample without trehalose during dehydration and shows primerdimer formation. Lane 1: $50 \mathrm{bp}$ DNA ladder, and lane 3 is negative control (no DNA template). $12 \mathrm{~B}$ shows $70 \mathrm{bp}$ amplified product after 28 days of dry preservation (lanes 4-6 corresponding to 10,15 and 20 $\%$ trehalose respectively). Lane 3 is positive control.

\section{CONCLUSIONS}

We have demonstrated the functionality of each subcomponent of the DEC system. We have demonstrated selective antibody capture for all target organisms directly from stool. We have shown pathogen lysis and nucleic acid extraction and capture on microfluidic cards. We have demonstrated PCR at the required sensitivity on microfluidic cards rapidly ( 8 minutes).
We have shown that reagents needed for immunocapture, lysis, and DNA amplification by PCR for pathogen detection can be stored in trehalose matrix in dry form and retain their activity upon reconstitution. In the future all of these components will be incorporated into a microfluidic platform, an on-chip device, for point-of care diagnostic systems. Finally, we have shown detection of amplification products on lateral flow strips. Current work is directed towards integration and field validation.

\section{REFERENCES}

[1] R. A. Guerrant, T. Van Gilder, T. S. Steiner, N. M. Thielman, L. Slutsker, R. V. Tauxe, T. Hennessy, P. M. Griffin, H. DuPont, R. B. Sack, P. Tarr, M. Neill, I. Nachamkin, L. B. Reller, M. T. Osterhol, M. L. Bennish, L. K. "Pickering. Infectious Disease Society of America Guidelines. Practice Guidelines for the Management of Infectious Diarrhea", Clinical Infectious Diseases, 32, 3 (2001) pp. 331-351.

[2] United States Food and Drug Administration Center for Food Safety and Applied Nutrition. Foodborne Pathogenic Microorganisms and Natural Toxins Handbook, January 2002.

[3] World Health Organization. "Guidelines for the Control of Epidemics due to Shigella dysenteriae type 1", WHO/CDR/95.1.

[4] T. J. Torok, R. V. Tauxe, R. P. Wise, J. R. Livengood, R. Sokolow, S. Mauvais, K. A. Birness, M. R. Skeels, J. R. Horan, L. R. Foster. "A large community outbreak of salmonellosis caused by intentional contamination of restaurant salad bars", Journal of American Medical Association, 278, 5 (1997) pp. 389-395.

[5] S. A. Kolavic, A. Kimura, S. L. Simons, L. Slutsker, S. Barth, C. E. Haley. "An outbreak of Shigella dysenteriae type 2 among laboratory workers due to intentional food contamination", Journal of American Medical Association, 278, 5 (1997) pp. 396-398.

[6] H. Karch, M. Bielaszewska. "Sorbitol-fermenting Shiga toxin-producing Escherichia coli O157:H(-) strains: epidemiology, phenotypic and molecular characteristics, and microbiological diagnosis", J Clin Microbiol. Review, 39, 6 (2001) pp. 2043-9.

[7] S. I. Tu, D. Patterson, C. Briggs, P. Irwin, L. Yu. "Detection of immunomagnetically captured Escherichia coli O157:H7 by antibody-conjugated alkaline phosphatase", Journal of Industrial Microbiology \& Biotechnology, 26, 6 (2001) pp. 345349.

[8] C. Gilbert, D. Winters, A. O'Leary, M. Slavik. "Development of a triplex PCR assay for the specific detection of Campylobacter jejuni, Salmonella spp., and Escherichia coli O157:H7", Molecular and Cellular Probes, 17, 4 (2003) pp. 135-138.

[9] NIAID Category A, B \& C Priority Pathogens. http://www.niaid.nih.gov/biodefense/bandc_priority.ht m (accessed October 2003). 
[10] Centers for Disease Control and Prevention. Summary of Notifiable Diseases-United States, (2001). Morbidity and Mortality Weekly Report, published May 2, 2003 for 2001/Vol. 50/No. 53.

[11] Centers for Disease Control and Prevention. "Outbreaks of Escherichia coli O157:H7 Infection and Cryptosporidiosis Associated with Drinking Unpasteurized Apple Cider-Connecticut and New York", October 1996. Morbidity and Mortality Weekly Report, 46, 01 (1997) pp. 4-8.

[12] A. Hatch, A. E. Kamholz, K. R. Hawkins, M. S. Munson, E. A. Schilling, B. H. Weigl, P. Yager. "A rapid diffusion immunoassay in a T-sensor", Nature Biotechnology, (invited review article), 19, 461 (2001).

[13] B. H. Weigl, P. Yager, "Microfluidic diffusion-based separation and detection", Science, 15, (1999) pp. 346-347.

[14] K. J. Halle, J. J. Li, M. S. Munson, J. Monteith, E. Guzman, S. Feather, J. Verba, Q. Porter, V. Kenning, A. E. Kamholz, B. H. Weigl, P. Saltsman, R. Bardell, P. Yager. "Capture and release concentration of bacteria using free-flow-zone electrophoresis." In: Harrison DJ and van den Berg A. eds. Micro Total Analysis Systems, Dordrecht: Kluwer Academic Publishers (2003).

[15]B. H. Weigl, C. J. Morris, N. Kesler, C. F. Battrell, R. L. Bardell. "Standard and high-throughput microfluidic disposables based on laminar fluid diffusion interfaces", In: Bornhop DJ, Dunn DA, Mariella DP Jr., et al, eds. Biomedical Nanotechnology Architectures and Applications, [Proceedings of SPIE v 4626] (2002) pp. 421-428.

[16] P. Jandik, B. H. Weigl, N. Kessler, J. Cheng, C. J. Morris, T. Schulte, N. Avdalovic. "Initial study of using laminar fluid diffusion interface for sample preparation in HPLC", Journal of Chromatography A. 954 (2002) pp. 33-40.

[17] B. H. Weigl, R. L. Bardell, T. H. Schulte, C. F. Battrell, J. Hayenga. "Design and rapid prototyping of thin-film laminate-based microfluidic devices", Biomedical Microdevices, 3, 4 (2001) pp. 267-274.

[18]B. H. Weigl. "New assays and separations based on laminar fluid diffusion interfaces-results from field trials for cell analysis, HTP screening, and medical diagnostics." In: Harrison DJ, van den Berg A, eds. Micro Total Analysis Systems, Dordrecht: Kluwer Academic Publishers (2002).

[19] C. F. Battrell, J. Hayenga, R. Bardell, C. Morris, P. Graham, N. Kesler, C. Lancaster, T. Schulte, B. H. Weigl, P. Saltsman, M. Shen. "Cell lysing and cytometry in an integrated microfluidic card system." In: Harrison DJ, van den Berg A, eds. Micro Total Analysis Systems, Dordrecht: Kluwer Academic Publishers (2002).

[20] T. H. Schulte, R. L. Bardell, B. H. Weigl. "Microfluidic technologies in clinical diagnostics", [invited review article]. Clinica Chimica Acta. 321, 1-2 (2002) pp. 1-10.
[21] B. H. Weigl, R. L. Bardell, C. R. Cabrera. "Lab-on-achip for drug development", [invited review article]. Advanced Drug Delivery Reviews, 55, 3 (2003) pp. 349-377.

[22] J. C. Gerdes. "Self-contained cartridge integrating nucleic acid extraction, amplification and lateral flow detection", J Clinical Ligand Assay, 20 (1997) pp. 70-72.

[23] K. Izutsu, S. Yoshioka, T. Terao. "Effect of mannitol crystallinity on the stabilization of enzymes during freeze-drying", Chem Pharm Bull, (Tokyo). 42, 1, (1994) pp. 5-8.

[24] J. H. Crowe, L. M. Crowe, J. F. Carpenter and C. Aurell Wistrom. "Stabilization of dry phospholipid bilayers and proteins by sugars", Biochem J, 242 (1987) pp. $1-10$ 